\title{
Interface conditions during mixed-mode phase transformations in metals
}

\author{
Richard M. Huizenga · Cornelis Bos • \\ Jilt Sietsma
}

Received: 7 July 2007/Accepted: 10 October 2007/Published online: 21 March 2008

(C) The Author(s) 2008

\begin{abstract}
A fast three-dimensional phase transformation model is formulated for the transformation from ferrite to austenite in low-carbon steel. The model addresses the parent microstructure, the nucleation behaviour of the new phase and the growth of the new phase. During the growth, the interface velocity of the ferrite grains is calculated using a mixed-mode growth model. The simulated transformation kinetics is compared with experimental kinetics for an $\mathrm{Fe}-\mathrm{C}-\mathrm{Mn}$ steel for four different cooling rates. In general, the model predicts the kinetics quite well. In addition, the mixed-mode character of the transformation is shown for the different cooling rates.
\end{abstract}

\section{Introduction}

Phase transformations in the solid state of metals take place in a two-step process: nucleation and growth. It is well known that nucleation in these transformations is primarily heterogeneous, taking place at lattice defects like interfaces or dislocations. But also, during growth the conditions at the interface are governing the process, influencing the jump frequency of atoms across the interface. The local free energy balance is determining this jump frequency,

\section{R. M. Huizenga $(\bowtie) \cdot C$. Bos · J. Sietsma}

Department of Materials Science and Engineering,

Delft University of Technology, Mekelweg 2, 2628 CD Delft,

The Netherlands

e-mail: r.m.huizenga@tudelft.nl

URL: http://www.mse.tudelft.nl

C. Bos

Netherlands Institute for Metals Research, Mekelweg 2,

2628 CD Delft, The Netherlands and thus the growth rate, involving the driving pressure for transformation and the activation energy for atomic jumps at the interface. The local free energy depends directly on the chemical compositions of the two phases at the interface, and on the temperature.

During the phase transformation of austenite to ferrite in low-carbon steel (typically $0.1 \mathrm{wt} \% \mathrm{C}$ ), the carbon will partition between ferrite and austenite, since the forming phase ferrite cannot contain more than $0.02 \mathrm{wt} \% \mathrm{C}$. The carbon concentration at either side of the interface directly determines the velocity of the interface and thus the kinetics of the transformation. Two processes play a major role in the phase transformation: the kinetics of the actual lattice transformation and the diffusion of the partitioning element, in this case carbon. While traditional models assume the kinetics to be controlled by either the lattice transformation (interface control [1]) or by carbon diffusion (diffusion control [2]), in this article a recently developed mixed-mode model $[3,4]$, taking into account both processes, is applied to an $\mathrm{Fe}-\mathrm{C}-\mathrm{Mn}$ steel and compared with experimental data.

\section{Model}

Three aspects are of primary importance for phase transformations: the parent microstructure, the nucleation behaviour of the new phase and the growth of the new phase. The way these aspects have been incorporated in the model is discussed in this section.

The parent microstructure (in this case austenite) is created from a three-dimensional random Voronoi tessellation, using periodic boundary conditions. The corners, edges and faces of the parent grains form the most favourable positions to act as nucleation sites for the 
new phase (in this case ferrite). Using this approach the spatial distribution of nuclei is according to the physical notion that applies to a real microstructure, and not random, as assumed in the widely applied Avrami like models [5-7]. This is relevant for the impingement of the grains and therefore for the transformation kinetics and the eventual grain size distribution. Furthermore, the temperature at which a nucleus becomes critical is defined separately for each nucleus, making it possible to simulate a spread in nucleation temperature [8]. A typical parent microstructure with several growing ferrite grains is shown in Fig. 1.

The ferrite nuclei grow spherically, with the interface velocity calculated according to [1]:

$v=M \Delta G$

with $M$ the interface mobility and $\Delta G$ the driving force. The mobility is calculated using an Arrhenius relation:

$M=M_{0} e^{-\frac{Q_{M}}{R T}}$

with $Q_{\mathrm{M}}$ the activation energy for Fe-atoms crossing the interface, $R$ the gas constant, $T$ the temperature and the pre-exponential factor $M_{0}$ given by:

$M_{0}=\frac{d^{4} v_{\mathrm{D}}}{k T} M^{*}$,

where $d$ is the jump distance across the interface, $v_{\mathrm{D}}$ the Debye frequency, $k$ the Boltzmann constant and $M^{*}$ can be used as a fitting parameter, indicating how much the actual mobility differs from the theoretical value.

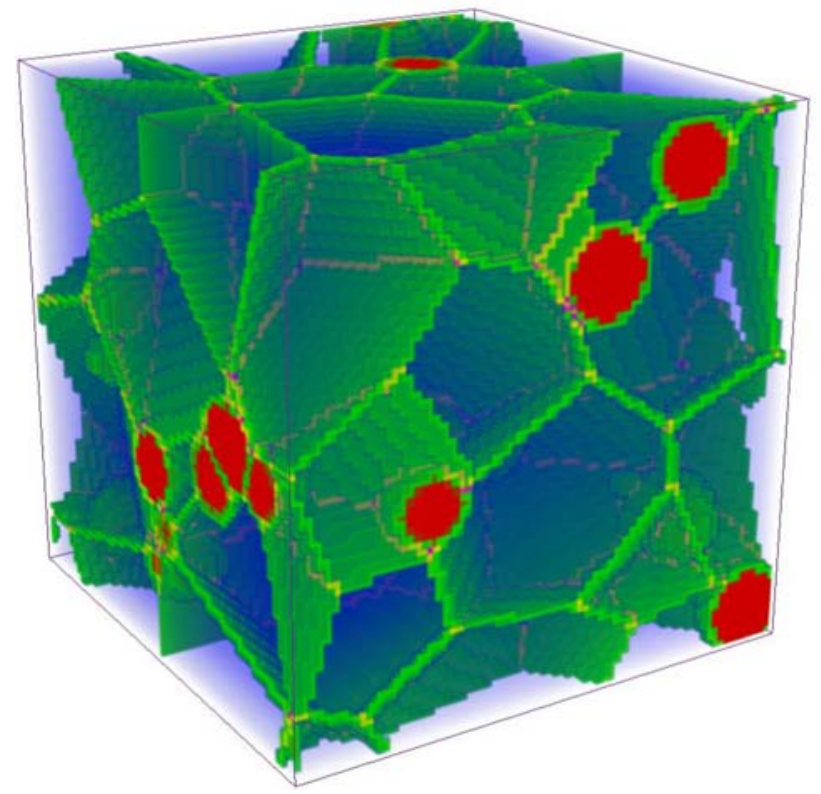

Fig. 1 Typical austenite microstructure showing internal corners, edges, faces and several ferrite grains (in red), which nucleated at the grain boundaries
The driving force $\Delta G$ is calculated from the difference of the chemical potentials between the phases, according to $[1]$ :

$\Delta G=X_{\mathrm{Fe}}^{\alpha}\left(\mu_{\mathrm{Fe}}^{\gamma}-\mu_{\mathrm{Fe}}^{\alpha}\right)+X_{\mathrm{Mn}}^{\alpha}\left(\mu_{\mathrm{Mn}}^{\gamma}-\mu_{\mathrm{Mn}}^{\alpha}\right)+X_{\mathrm{C}}^{\alpha}\left(\mu_{\mathrm{C}}^{\gamma}-\mu_{\mathrm{C}}^{\alpha}\right)$,

with $X_{\mathrm{j}}^{\alpha}$ the atomic concentration $(j=\mathrm{Fe}, \mathrm{Mn}, \mathrm{C})$ in ferrite and $\mu_{j}^{\varphi}$ the chemical potential at the interface for element $j$ in phase $\varphi$ (where $\alpha$ is ferrite, $\gamma$ is austenite).

The chemical potential of an element in a phase depends on the temperature and the local composition in the phase. Since the diffusion of carbon is much faster than the diffusion of manganese, we assume that only the carbon partitions and the manganese atoms do not redistribute. This means that the ratio $X_{\mathrm{Fe}} / X_{\mathrm{Mn}}$ stays constant in the phases during the transformation (so-called para-equilibrium). The system can therefore be regarded as a binary $\mathrm{M}-\mathrm{C}$ system, with $\mathrm{M}$ representing the metals $\mathrm{Fe}$ and $\mathrm{Mn}$. The chemical potential of $\mathrm{M}$ can be written as:

$\mu_{\mathrm{M}}^{\varphi}=Y_{\mathrm{Fe}}^{\varphi} \mu_{\mathrm{Fe}}^{\varphi}+Y_{\mathrm{Mn}}^{\varphi} \mu_{\mathrm{Mn}}^{\varphi}$,

with $Y_{\mathrm{Fe}}^{\varphi}$ the relative fraction of Fe: $Y_{\mathrm{Fe}}^{\varphi}=X_{\mathrm{Fe}}^{\varphi} / X_{\mathrm{M}}^{\varphi}$ and $Y_{\mathrm{Mn}}^{\varphi}$ the relative fraction of Mn: $Y_{\mathrm{Mn}}^{\varphi}=X_{\mathrm{Mn}}^{\varphi} / X_{\mathrm{M}}^{\varphi}$, where $X_{\mathrm{M}}^{\varphi}=$ $X_{\mathrm{Fe}}^{\varphi}+X_{\mathrm{Mn}}^{\varphi}$ in a given phase $\varphi$.

The driving force can now be rewritten as:

$\Delta G=X_{\mathrm{M}}^{\alpha}\left(\mu_{\mathrm{M}}^{\gamma}-\mu_{\mathrm{M}}^{\alpha}\right)+X_{\mathrm{C}}^{\alpha}\left(\mu_{\mathrm{C}}^{\gamma}-\mu_{\mathrm{C}}^{\alpha}\right)$

This binary system is actually an intersection of the threedimensional Gibbs surfaces at the plane where the ratio $X_{\mathrm{Fe}} / X_{\mathrm{Mn}}$ is constant, leading to a two-dimensional Gibbs free energy diagram. This approach is similar as described by Gamsjäger et al. [9]. A graphical illustration of the driving force is shown in Fig. 2.

As can be seen in Fig. 2, the driving force is directly related to the carbon concentration in austenite at the interface. The carbon concentration in ferrite is assumed to be the equilibrium concentration as given by the Gibbscurves, which is very low. When the ferrite grows, the carbon is expelled into the austenite, causing an increase of the carbon concentration in austenite, in particular at the interface. For this concentration two important processes play a role simultaneously: (1) the velocity of the interface (pushing more carbon into austenite) and (2) the diffusion of carbon away from the interface into the austenite matrix. Determining the exact interface concentration is therefore not straightforward.

A fast analytical model of the interface concentration was described by Sietsma and van der Zwaag [3] where both the mobility of the interface and the diffusion of carbon in austenite were taken into account. In this mixedmode model, the carbon concentration profile in austenite was approximated using a linear profile. This approach was recently refined using an exponential concentration profile in three-dimensional and taking into account soft 


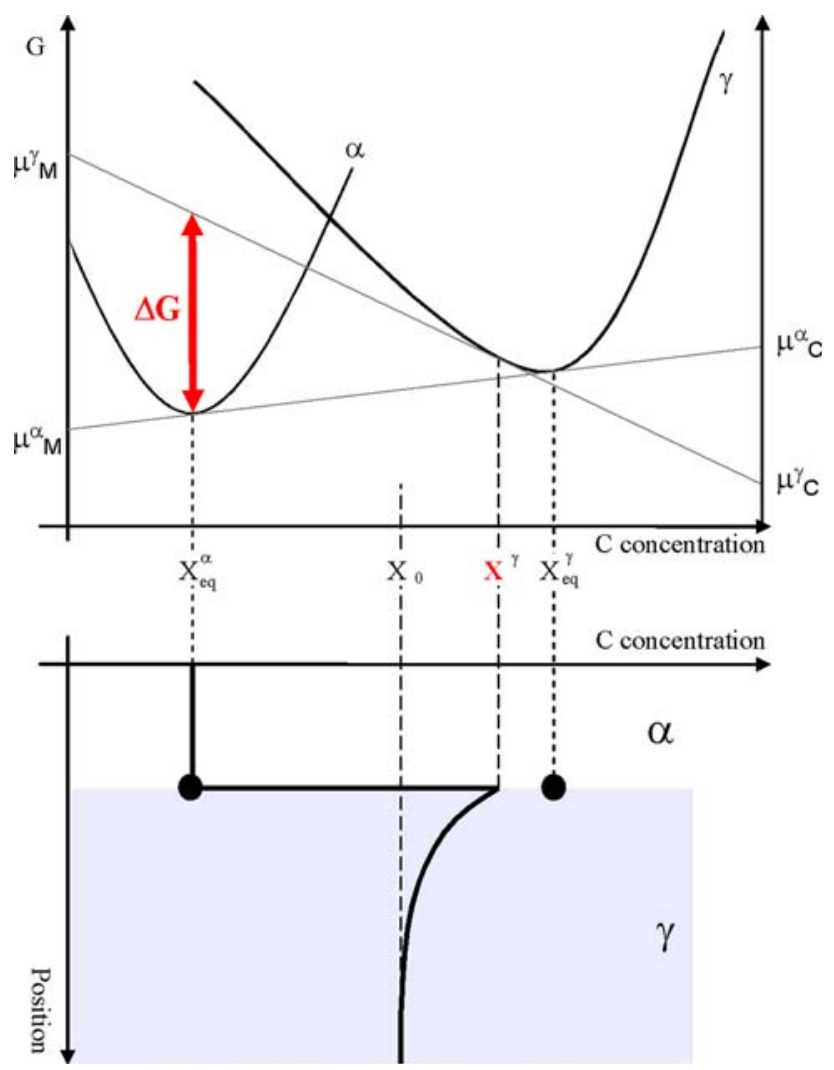

Fig. 2 Schematic illustration of the driving force $(\Delta G)$ using Gibbs free energy curves (top part of the figure) in relation to the Cconcentration profile (bottom part, profile is rotated $90^{\circ}$ ). $X^{\gamma}$ indicates the concentration at the interface, $X_{\mathrm{eq}}$ indicates the equilibrium compositions and $X_{0}$ is the overall concentration

impingement as well [4]. The exponential concentration profile used in that model is given by:

$X=X_{0}+\left(X^{\gamma}-X_{0}\right) \exp \left(-\frac{z}{z_{0}}\right)$,

with $X_{0}$ the bulk concentration, $X^{\gamma}$ the interface concentration, $z_{0}$ a parameter determining the width of the profile, which is determined by the carbon mass balance, and $z$ the distance from the interface in austenite. For the carbon diffusion in austenite the model uses:

$D=D_{0} e^{-\frac{Q_{\mathrm{D}}}{R T}}$,

with $D_{0}$ the pre-exponential constant and $Q_{\mathrm{D}}$ the activation energy for carbon diffusion in austenite.

This refined mixed-mode growth model is incorporated in the three-dimensional transformation model presented in this article. At each time step and for each ferrite grain, the carbon concentration profile in austenite is calculated, from which the interface concentration is determined. If the mobility is high and the diffusivity is low, the transformation is almost diffusion controlled, and the interface concentration will be close to $X^{\gamma}$ eq. In the opposite case, the transformation is nearly interface controlled, and the interface concentration will be close to the homogeneous concentration in austenite. The interface concentration in austenite gives the driving force and thus the velocity of the interface, from which the new radius for each grain is calculated. Finally, the ferrite fraction is calculated by numerical integration, taking into account the impingement of the grains (overlapping volumes of overlapping spheres are counted only once).

The calculations for this model are very fast (in the order of minutes to half an hour), especially compared to phase-field modelling [10], where simulation times of days or weeks for three-dimensional-simulations are not uncommon.

\section{Simulation}

The model is applied for an Fe-0.1 C-0.49 Mn wt\% steel, which is cooled from $900{ }^{\circ} \mathrm{C}$ to temperatures below the Ae1-temperature using four different cooling rates: 0.05 , $0.3,10$ and $60 \mathrm{~K} / \mathrm{s}$. The model is compared with experimental data from Kop [11]. An example of the simulated austenite to ferrite transformation is shown in Fig. 3.

\section{Nucleation}

The nucleation density is determined from the final ferrite grain size [11]. The four different cooling rates result in quite different nucleation behaviour, which is included in the simulations in the following way. For the $0.05 \mathrm{~K} / \mathrm{s}$ cooling rate a nucleation range of $0-30 \mathrm{~K}$ below the Ae3temperature is used [8]. For a cooling rate of $0.3 \mathrm{~K} / \mathrm{s}$ a slightly larger range of $0-40 \mathrm{~K}$ is applied. In both cases nuclei appear on corners and edges. For the cooling rate of $10 \mathrm{~K} / \mathrm{s}$ the nucleation range is increased to $0-60 \mathrm{~K} \mathrm{[12]}$ and for the $60 \mathrm{~K} / \mathrm{s}$ cooling rate a range of $0-150 \mathrm{~K}$ is used. In the last two cases, it is assumed that the nuclei appear on corners, edges and faces. In all cases a constant nucleation rate is applied, so the number of nuclei increases linearly with decreasing temperature.

\section{Growth}

The parameters used for the simulation are listed in Table 1 , where the only fitting parameter used is $M^{*}$. The simulation domains for the higher cooling rates of 10 and $60 \mathrm{~K} / \mathrm{s}$ have half the dimensions of the lower cooling rates to reduce the calculation time. Since the nucleation density is much higher for high cooling rates, the resulting number of nuclei is on the same order of magnitude. 
Fig. 3 Typical simulated microstructure of ferrite (red) growing into an austenite (blue) parent phase
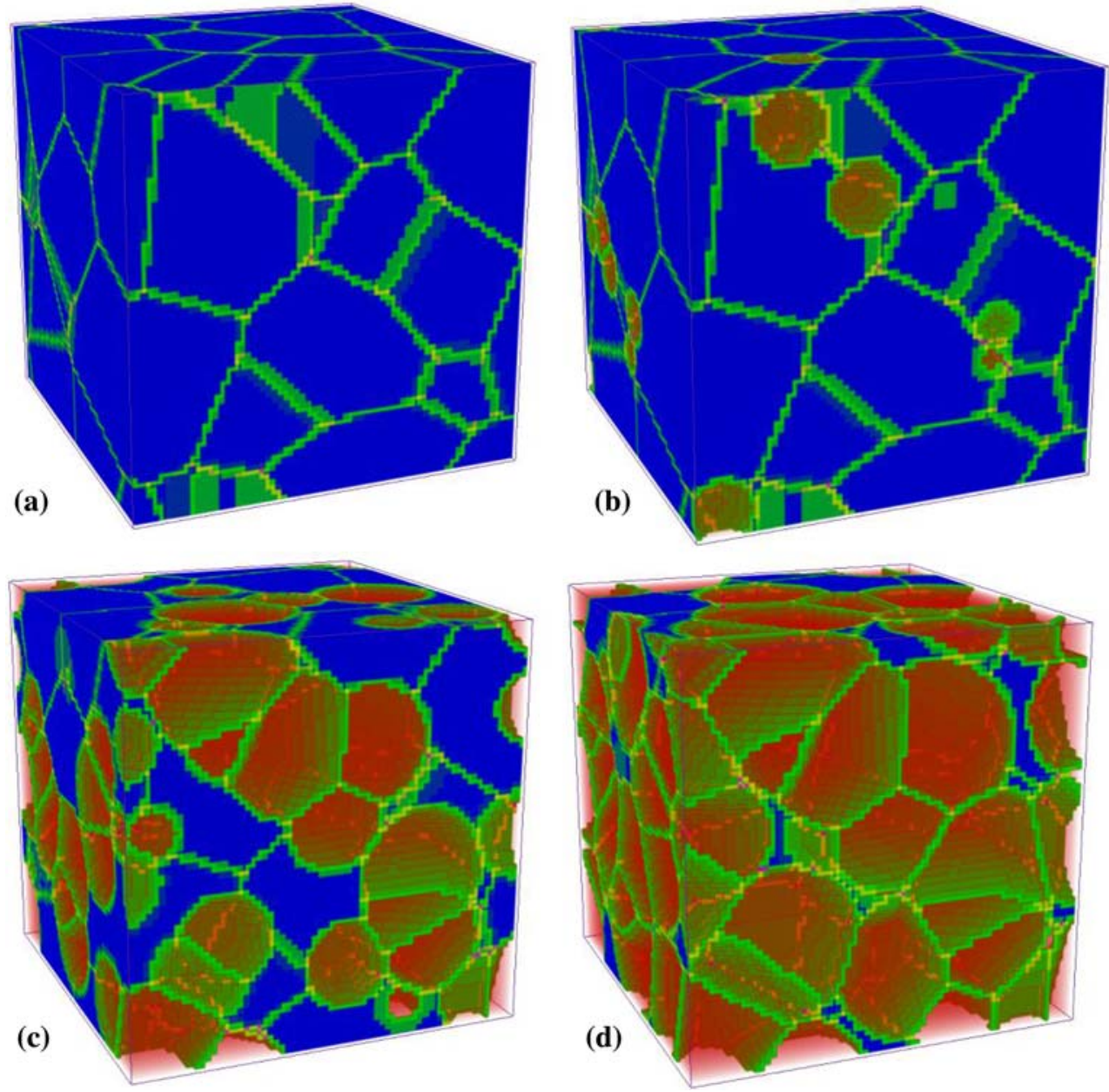

Table 1 Simulation parameters

$$
\begin{aligned}
& 1.50 \times 10^{-5}[14] \\
& 142.1[14] \\
& 140[13] \\
& 6.15 \times 10^{13} \\
& 2.50 \times 10^{-10}
\end{aligned}
$$

\begin{tabular}{lllll}
\hline & \multicolumn{4}{l}{ Cooling rates $\left[\mathrm{K} \mathrm{s}^{-1}\right]$} \\
\cline { 2 - 5 } & 0.05 & 0.3 & 10 & 60 \\
\hline Domain dimension $(\mu \mathrm{m})$ & $66 \times 66 \times 66$ & $66 \times 66 \times 66$ & $33 \times 33 \times 33$ & $33 \times 33 \times 33$ \\
Number of austenite grains & 216 & 216 & 27 & 27 \\
Nucleation range $(\mathrm{K})$ & $0-30$ & $0-40$ & $0-60$ & $0-150$ \\
Nucleation density $\left(10^{13} \mathrm{~m}^{-3}\right)$ & 13 & 30 & 195 & 462 \\
Number of ferrite nuclei & 37 & 86 & 70 & 166 \\
Mobility factor $M^{*}$ & 0.08 & 0.08 & 0.13 & 2 \\
\hline
\end{tabular}

From experiments done by Kop [11], it can be concluded that the actual Ae3-temperature for this steel (under para-equilibrium conditions) is about $15 \mathrm{~K}$ lower than calculated by thermodynamic software. Therefore, the equilibrium conditions used in the simulations are shifted accordingly (the Ae3-temperature from thermodynamic databases is $1,117 \mathrm{~K}$; consequently $1,102 \mathrm{~K}$ is used in simulations). 


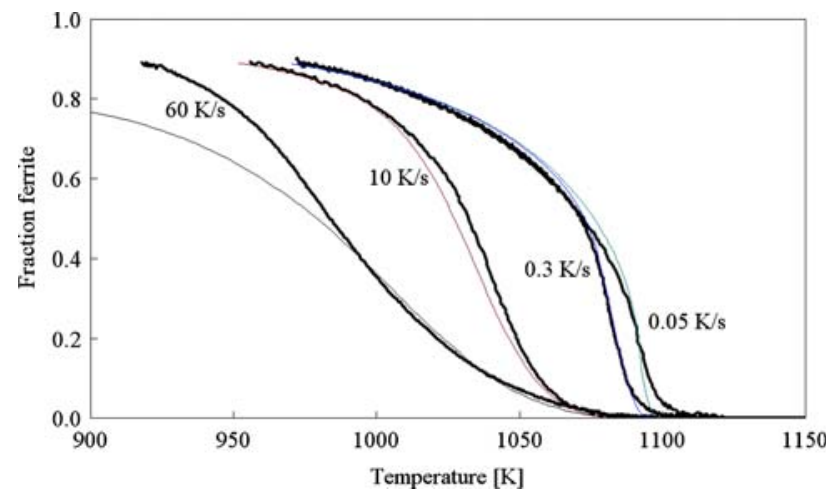

Fig. 4 Experimental fraction curves (thick lines) as function of temperature compared with simulated fraction curves (thin lines)

\section{Results and discussion}

Fraction curves

In general, the ferrite fraction curves show a very good agreement with the experimental data for the different cooling rates (see Fig. 4). Only at the start of the curves for the low cooling rates, the experimental data shows some ferrite forming at higher temperatures. This is probably caused by an inhomogeneous Mn distribution and temperature gradients in the sample, which give rise to locally different conditions. For the $10 \mathrm{~K} / \mathrm{s}$ cooling rate, the simulation follows the experiment quite closely for the whole transformation. For the $60 \mathrm{~K} / \mathrm{s}$ cooling rate the model correctly simulates the experiment up to a ferrite fraction of 0.5 and then continues at a slower rate, due to a low mobility in the model at low temperature. It is possible that in this case also more nuclei are appearing at the end of transformation, but the exact nucleation behaviour is currently unknown.

\section{Interface concentration}

To show the mixed-mode character of the transformation for the four different cooling rates, the carbon concentration at the interface in austenite of one of the nuclei is
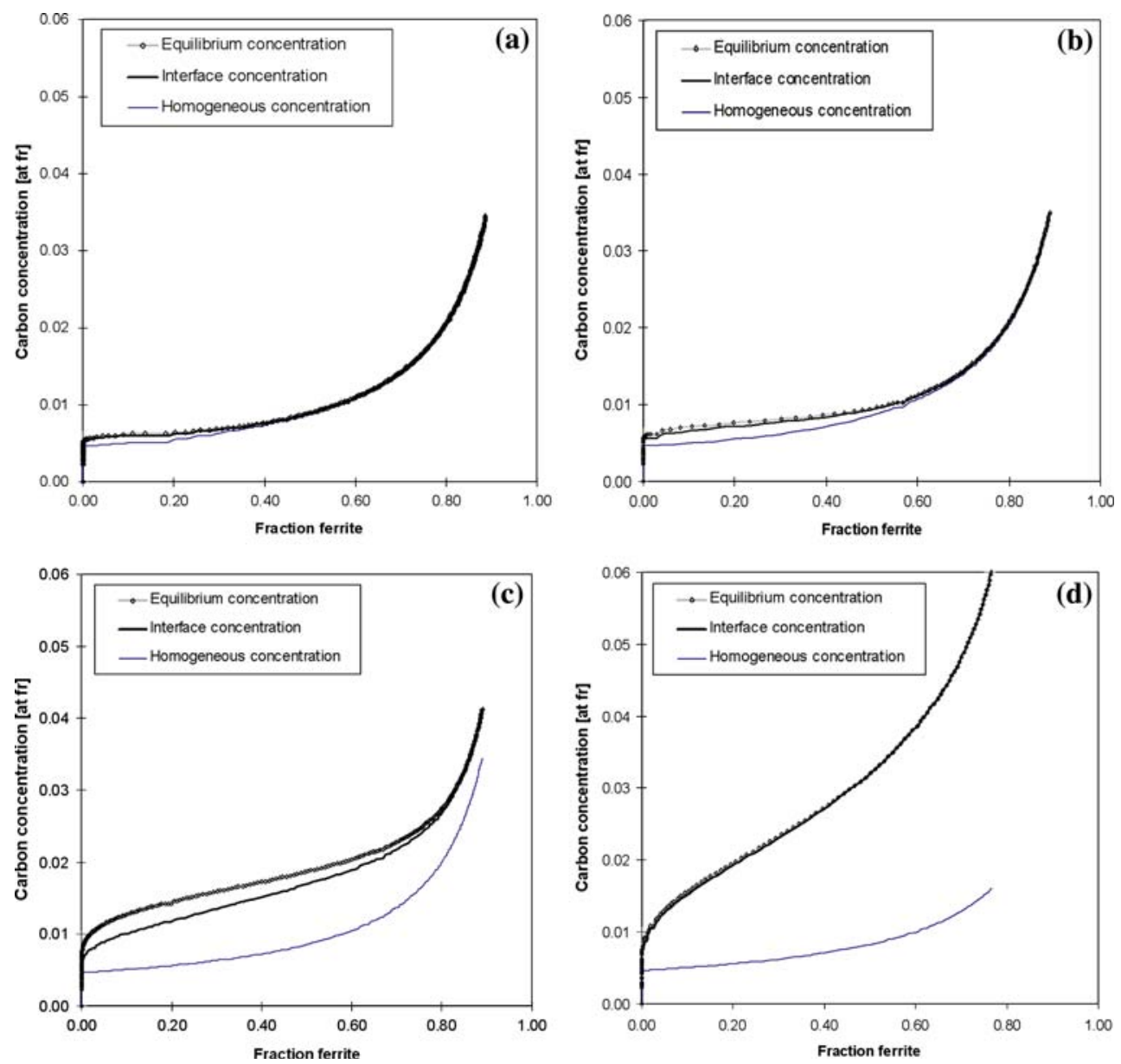

Fig. 5 Carbon concentrations at the interface in austenite as function of fraction ferrite, compared with equilibrium and homogeneous concentrations in austenite for different cooling rates: (a) 0.05 , (b) 0.3 , (c) 10 , (d) $60 \mathrm{~K} / \mathrm{s}$ 


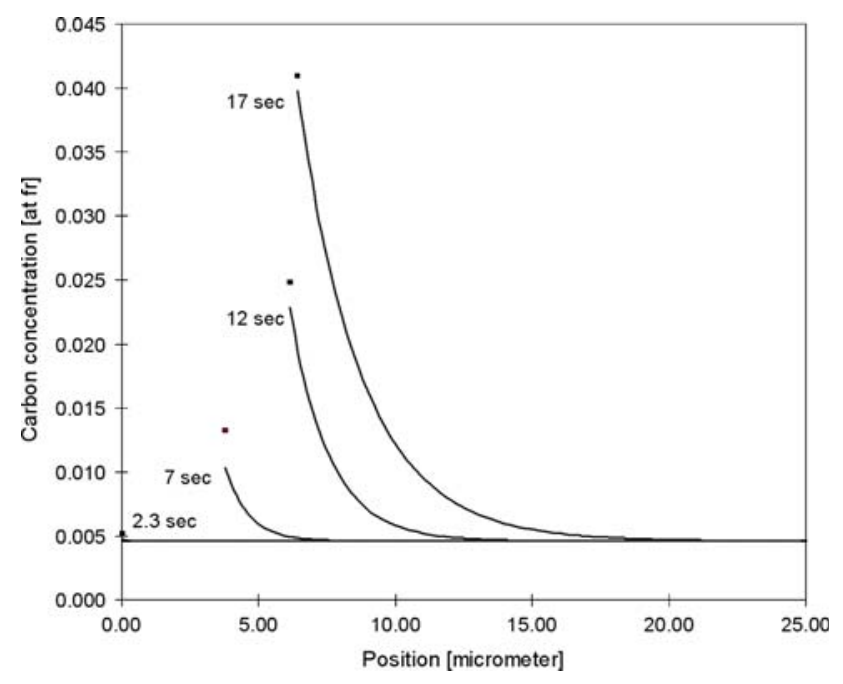

Fig. 6 The development of the carbon concentration profile in austenite over time (for $10 \mathrm{~K} / \mathrm{s}$ cooling rate, without soft impingement effect). The squares above the profiles indicate the equilibrium concentrations

compared with the equilibrium and homogeneous concentration (Fig. 5).

For the very low cooling rate of $0.05 \mathrm{~K} / \mathrm{s}$ the equilibrium and the homogeneous carbon concentration are quite close, since the transformation develops almost exactly according to the temperature-dependent equilibrium phase fractions. Consequently, there is not much difference between diffusion- and interface-controlled growth. After $40 \%$ transformed ferrite, the transformed fraction can no longer be distinguished from the equilibrium fraction.

For the $0.3 \mathrm{~K} / \mathrm{s}$ cooling rate the carbon interface concentration is slightly below the equilibrium concentration in austenite, and therefore the transformation is close to diffusion-controlled. After $60 \%$ ferrite, the transformation follows equilibrium.

For the $10 \mathrm{~K} / \mathrm{s}$ cooling rate the transformation clearly shows a mixed-mode character: the carbon concentration is well below the equilibrium concentration during most of the transformation, but is also far above the homogeneous concentration. Therefore the transformation is between diffusion- and interface-controlled. The concentration profiles of the first appearing nucleus during the transformation are shown in Fig. 6 for different stages of the transformation. As the ferrite grows, the carbon is pilling up at the austenite interface, but is not reaching the equilibrium concentration for most of the transformation. These profiles are an important result of the modelling, since they indicate the degree of soft impingement (overlap of different diffusion fields) during the phase transformation, as well as resulting concentration gradients in any retained austenite.
For the cooling rate of $60 \mathrm{~K} / \mathrm{s}$ the interface concentration is right from the start just below the equilibrium concentration, indicating mainly diffusion-controlled growth. However, the agreement with the experimental data is rather poor in this case (see Fig. 4), which has possibly led to a high value being chosen for the mobility.

\section{Conclusions}

The presented model allows very fast simulations of partitioning phase transformations in three-dimensional space, and is shown to be able to simulate the austenite to ferrite transformation kinetics quite well for $0.05,0.3$ and $10 \mathrm{~K} / \mathrm{s}$ cooling rates. In the case of $60 \mathrm{~K} / \mathrm{s}$ cooling rate, the model follows experiment until about half the transformation. The model does not a priori assume diffusion control or interface control for the growth, but the character of the transformation is a result of the modelling.

The mixed-mode character of the transformation is seen clearly in the case of $10 \mathrm{~K} / \mathrm{s}$ where the carbon interface concentration is between the homogeneous and equilibrium concentration.

Open Access This article is distributed under the terms of the Creative Commons Attribution Noncommercial License which permits any noncommercial use, distribution, and reproduction in any medium, provided the original author(s) and source are credited.

\section{References}

1. Christian J (1981) The theory of phase transformations in metals and alloys. Pergamon Press, Oxford

2. Zener C (1949) J Appl Phys 20:950

3. Sietsma J, van der Zwaag S (2004) Acta Mater 52:4143

4. Bos C, Sietsma J (2007) Scripta Materialia 57:1085

5. Johnson WA, Mehl RF (1939) Trans AIME 135:416

6. Avrami M (1939) J Chem Phys 7:1103

7. Kolmogorov AN (1937) Izv Akad, Nauk, SSSR, Ser Mat 3:355

8. Offerman SE, van Dijk NH, Sietsma J, Grigull S, Lauridsen EM, Margulies L, Poulsen HF, Rekveldt MT, van der Zwaag S (2002) Science 298:1003

9. Gamsjäger E, Fischer FD, Svoboda J (2003) ASME J Eng Mater Technol 125:22

10. Tiaden J, Nestler B, Diepers HJ, Steinbach I (1998) J Phys D Appl Phy 115:73

11. Kop T (2000) A dilatometric study of the austenite/ferrite interface mobility, Ph.D thesis, Delft University of Technology

12. Mecozzi MG (2007) Phase field modeling of the austenite to ferrite transformation in steels, Ph.D thesis, Delft University of Technology

13. Krielaart GP, van der Zwaag S (1998) Mater Sci Techn 14:10

14. Weast RC (1989) Handbook of chemistry and physics. CRC Press Inc., Boca Raton 